\title{
3 Improved growth and morphological plasticity of Haloferax volcanii
}

4

5

6

7

8

9

$10{ }^{1}$ The iThree Institute, University of Technology Sydney, New South Wales, 2007, 11 Australia.

$12{ }^{2}$ Department of Biology, University of Pennsylvania, Philadelphia, PA 19104, USA.

13

14

15

16

17

18 Keywords: pleomorphology, trace elements, cytoskeleton, growth media,

19 halophile, archaea

20

*Address correspondence to: Iain.Duggin@uts.edu.au
19 halophile, archaea
Running title: Morphological plasticity of $H$. volcanii 


\section{Abstract}

22 Some microbes display pleomorphism, showing variable cell shapes in a single 23 culture, whereas others differentiate to adapt to changed environmental conditions.

24 The pleomorphic archaeon Haloferax volcanii commonly forms discoid-shaped 25 ('plate') cells in culture, but may also be present as rods, and can develop into motile 26 rods in soft agar, or longer filaments in certain biofilms. Here we report improvement 27 of $H$. volcanii growth in both semi-defined and complex media by supplementing 28 with eight trace-element micronutrients. With these supplemented media, transient 29 development of plate cells into uniformly-shaped rods was clearly observed during 30 the early log phase of growth; cells then reverted to plates for the late log and 31 stationary phases. In media prepared with high-purity water and reagents, without 32 supplemental trace elements, rods and other complex elongated morphologies 33 ('pleomorphic rods') were observed at all growth stages of the culture; the highly34 elongated cells sometimes displayed a substantial tubule at one or less frequently both 35 poles, as well as unusual tapered and highly-curved forms. Polar tubules were 36 observed forming by initial mid-cell narrowing or tubulation, causing a dumbbell-like 37 shape, followed by cell division towards one end. Formation of the uniform early-log 38 rods, as well as the pleomorphic rods and tubules were dependent on the function of 39 the tubulin-like cytoskeletal protein, CetZ1. Our results have revealed the remarkable 40 morphological plasticity of $H$. volcanii cells in response to multiple culture 41 conditions, and should facilitate the use of this species in further studies of archaeal 42 biology. 


\section{$44 \quad$ Introduction}

45 Archaea exhibit some of the most diverse and unusual microbial cell morphologies.

46 Cell shapes range from rods and cocci to striking triangles and squares. However,

47 little is known about the mechanisms and environmental cues that dictate archaeal cell morphology, or the regulation of transitions between cell morphologies. The model haloarchaeon, Haloferax volcanii, when first isolated, was described as mainly diskshaped cells, with cell shape and size varying significantly (1). In routine liquid cultures of $H$. volcanii, both pleomorphic discoid (plate) and rod cell morphologies may be observed, occasionally with more unusual shapes (2-4).

53 The conditions that influence $H$. volcanii cell shapes and the relative abundance of the 54 distinct types are not well understood, and specific signals have not been identified. In $H$. volcanii biofilms, substantial elongation (filamentation) has been observed in a subpopulation of cells (5). Furthermore, $H$. volcanii forms motile rods in soft-agar, where rods are observed at the forefront of expanding colonies of swimming cells (6). The tubulin-like cytoskeletal protein CetZ1 is required for rod-formation, suggesting a connection between morphology and motility that may be expected based on improved hydrodynamics or directional movement of rods $(4,6)$. Conversely, the peptidase archaeosortase A (ArtA) and phosphatidylethanolamine biosynthesis enzymes PssA and PssD, which are required for the C-terminal processing and covalent lipid attachment of several $H$. volcanii surface proteins including the surface (S-layer) glycoprotein, are required for effective and stable plate-shaped cell formation $(7,8)$.

To better understand cell shape switching and the significance of the distinct shapes, we aimed to define conditions that can be robustly used to study these processes. We determined that inclusion of a trace elements (TE) solution in both complex and semidefined culture media substantially improves culture and cell shape reproducibility for this species. In the new media, early-log rod formation was also more reproducible,

71 while the pleomorphic rods and other shapes seen in cultures lacking TE were absent.

72 We further defined culture conditions and characterized these CetZ1-dependent 73 morphological changes during both TE-depletion and early-log rod formation in 74 replete cultures. These conditions may serve as experimental models for studies of 
bioRxiv preprint doi: https://doi.org/10.1101/2020.05.04.078048; this version posted December 1, 2020. The copyright holder for this preprint (which was not certified by peer review) is the author/funder. All rights reserved. No reuse allowed without permission.

75 morphological development in archaea. 
76

77

78

79

80

81

82

83

84

85

86

87

88

89

90

91

92

93

94

95

96

97

98

99

100

101

102

103

104

105

106

\section{Methods}

\section{Archaeal strains}

Haloferax volcanii H26 ( containing the pHV2-based plasmids pTA962 or pTA963 (9) as indicated, were used as wild-type strains. H. volcanii ID59 (6), transformed with pTA962 (i.e. ID113), was the cetZ1 knockout strain used (based on H98). Attempts to verify the roles of CetZ1 by plasmid-based expression of wild-type cetZl in the $\Delta c e t Z 1$ strain (ID59) were unsuccessful, due to polar effects on a downstream gene (unpublished results), so we expressed cetZ1.E218A (a dominant-inhibitory mutant) to confirm the involvement of cetZ1, by using H98 + pTA962-cetZ1.E218A, as described previously (6).

\section{Culture media and general growth conditions}

Media were based on the Hv-Ca and Hv-YPC media commonly used for H. volcanii (10). In this study, we used defined sources of all media reagents, which we found to be important for reproducibility. However, the data reporting the initial observation of early-culture rod development (Fig. 1) were obtained with the original recipe for $\mathrm{Hv}$ Ca (10). For solid media, Bacteriological Agar (Oxoid LP0011) (10 g/L), was dissolved in the volume of water required for the medium by heating in a microwave oven, before mixing with pre-heated concentrated stock solutions of macronutrients and buffered salt water solutions (listed below) and autoclaving, followed by addition of trace elements or heat-sensitive reagents. Water was obtained from an ultrapure (18.2 MOhm.cm) water purification system (Sartorius).

Hv-Ca medium was prepared similarly to that described previously (10), however, in all experiments except Fig. 1, Hv-Ca medium also contained an additional vitamin solution. Hv-Ca contained: $144 \mathrm{~g} / \mathrm{L} \mathrm{NaCl}$ (Sigma S6191), $21 \mathrm{~g} / \mathrm{L} \mathrm{MgSO} \bullet_{4} \cdot 7 \mathrm{H}_{2} \mathrm{O}$ (Sigma M1880), $18 \mathrm{~g} / \mathrm{L} \mathrm{MgCl}_{2} \bullet 6 \mathrm{H}_{2} \mathrm{O}$ (Sigma M2393), $4.2 \mathrm{~g} / \mathrm{L} \mathrm{KCl}$ (Sigma P5405), 12 mM Tris-HCl (pH 7.4) (Sigma RDD008, buffered with AR grade $\mathrm{HCl}$ ), $3 \mathrm{mM}$ $\mathrm{CaCl}_{2}$ (Sigma C5670), and $5 \mathrm{~g} / \mathrm{L}$ Casamino Acids (Oxoid LP0041, prepared initially as a 10x stock solution). The medium was then autoclaved. Once cool, a filtersterilized vitamin concentrated stock solution (1000x), containing $1 \mathrm{~g} / \mathrm{L}$ thiamine (Sigma T1270) and $0.1 \mathrm{~g} / \mathrm{L}$ biotin (Sigma B4639), was added (1 mL per L of medium). Buffered salt water (BSW) was prepared as the buffered salts solution 
107 described above, i.e. Hv-Ca medium (18\% w/v total salts), omitting the Casamino

108 acids and vitamins. BSW was prepared as a concentrated (30\% w/v salts) stock

109 solution.

110 To prepare Hv-Cab medium, a trace-elements concentrated stock solution (100x) was 111 added (10 $\mathrm{mL}$ per $\mathrm{L})$ to the $\mathrm{Hv}-\mathrm{Ca}$ medium after autoclaving and cooling (including 112 vitamins). The 100x trace-elements stock solution contained: $5 \mathrm{~g} / \mathrm{L} \mathrm{Na} \mathrm{NDTA}_{2} \cdot 2 \mathrm{H}_{2} \mathrm{O}$ 113 (Sigma E1644), $0.8 \mathrm{~g} / \mathrm{L} \mathrm{FeCl}_{3}$ (Sigma 157740), $0.05 \mathrm{~g} / \mathrm{L} \mathrm{ZnCl}_{2}$ (Sigma 793523), 0.01 $114 \mathrm{~g} / \mathrm{L} \mathrm{CuCl}_{2}$ (Sigma 751944), $0.01 \mathrm{~g} / \mathrm{L} \mathrm{CoCl}_{2}$ (Sigma 232696), $0.01 \mathrm{~g} / \mathrm{L} \mathrm{H}_{3} \mathrm{BO}_{3}$ (Sigma 115 B6768), $1.6 \mathrm{~g} / \mathrm{L} \mathrm{MnCl}_{2}$ (Sigma 328146), $0.01 \mathrm{~g} / \mathrm{L} \mathrm{NiSO}_{4}$ (Sigma 656895), and 0.01 $116 \mathrm{~g} / \mathrm{L} \mathrm{Na}_{2} \mathrm{MoO}_{4} \cdot 2 \mathrm{H}_{2} \mathrm{O}$ (Sigma M1003); the $\mathrm{pH}$ of the solution was adjusted to 7.0 with $117 \mathrm{NaOH}$ (AR grade, Sigma), and then the obvious brown precipitate that formed on 118 neutralization was removed by $0.2 \mu \mathrm{m}$ sterile filtration; stock solutions were stored at 119 room temperature in sterile aliquots.

120 Hv-YPC medium (10) was prepared as per the Hv-Ca medium above, except the 121 supplementary vitamins were omitted, the Casamino acids was used at a concentration 122 of $1 \mathrm{~g} / \mathrm{L}$, and $5 \mathrm{~g} / \mathrm{L}$ Yeast Extract (Oxoid LP0021) and $1 \mathrm{~g} / \mathrm{L}$ Peptone (Oxoid LP0037) 123 were included (initially dissolved as a 10x stock solution). To prepare "Hv-YPCab" 124 medium, the 100x trace-elements stock solution was added (10 mL per L of medium) 125 to Hv-YPC after autoclaving and cooling.

126 Most cultures $(5 \mathrm{~mL})$ were grown in sterile $50 \mathrm{~mL}$ Falcon tubes, incubated at $45^{\circ} \mathrm{C}$ 127 with shaking at $200 \mathrm{rpm}$ in a GFL 1092 rotary-shaking water bath (the tube's lid was 128 maintained loosened) unless otherwise indicated. H. volcanii was initially grown on 129 solid medium followed by continuous growth in liquid medium (diluted once per 130 day), to maintain steady logarithmic growth prior to sampling, which was only done 131 after the culture had been growing steadily in continuous log-phase for at least 2 days, 132 unless otherwise indicated. For obtaining growth curves in microtitre plates, cultures 133 established as above at $45^{\circ} \mathrm{C}$ were used to prepare a culture with a starting $\mathrm{OD}_{600}$ of 1340.1 in the indicated Hv-YPC based media, with or without TEs as indicated. Growth 135 at $42^{\circ} \mathrm{C}$ with $200 \mathrm{rpm}$ shaking was then monitored in a Tecan Spark microtiter plate 136 spectrophotometer (30 min reading time intervals).

137 Time-course studies of rod development 
138 In order to obtain a standardized way of observing rod cells, and analyzing rod-

139 development, time-course studies of the early stages of liquid culture growth were 140 carried out (Fig. 4). The H. volcanii strain was streaked onto an agar plate made from 141 the same medium as chosen for the assay (e.g. Hv-YPCab), and then the plate was 142 incubated in sealed humidified bag at $45^{\circ} \mathrm{C}$ for 4 days. Colonies were collected from 143 the surface with a microbiological loop, and resuspended in $5 \mathrm{~mL}$ of liquid medium, 144 sufficient to give an $\mathrm{OD}_{600}>0.05$. The $\mathrm{OD}_{600}$ was immediately adjusted to 0.05 by 145 dilution with fresh pre-warmed medium, marking the start of the time-course. 146 Samples were withdrawn as required for microscopy or other analyses (Fig. 4). In 147 some experiments, cells were obtained directly from a mid-log liquid culture (Fig. 148 S1B).

149 To observe trace element limitation over a time-course (Fig. 4), cultures were first 150 grown into steady $\log$ phase in $\mathrm{Hv}-\mathrm{Cab}$ medium at $45^{\circ} \mathrm{C}$, with shaking at $200 \mathrm{rpm}$ (5 $151 \mathrm{~mL}$ culture). A sample of the culture was withdrawn and centrifuged at 5,000 rpm for $1525 \mathrm{~min}$ in an Eppendorf microcentrifuge. The supernatant was discarded and the cells 153 were then washed by re-suspending the pellet in $1 \mathrm{~mL}$ of $18 \% \mathrm{BSW}$. The wash was 154 repeated three times and then the cells were finally re-suspended in $0.1 \mathrm{~mL} 18 \% \mathrm{BSW}$ 155 and then spread onto Hv-Ca agar plate(s). The plate(s) were placed into a plastic bag 156 and incubated at $45^{\circ} \mathrm{C}$ for 4 days. $5 \mathrm{~mL}$ of liquid $\mathrm{Hv}-\mathrm{Ca}$ was added to the plate 157 surface and the cells were re-suspended, avoiding disturbing the agar. The $\mathrm{OD}_{600}$ was 158 adjusted to 0.05 with $\mathrm{Hv}-\mathrm{Ca}$ medium, to start the time course (incubation was 159 continued at $45^{\circ} \mathrm{C}$ at $\left.200 \mathrm{rpm}\right)$.

160 Microscopy

161 Samples were prepared by mounting a $2 \mu \mathrm{l}$ volume of culture (concentrated where 162 necessary by centrifugation and gentle resuspension) onto a slide prepared with a $163 \sim 170 \mu \mathrm{m}$ thick, 1\% agarose pad containing 18\% BSW (6). Most phase-contrast 164 images were acquired with a Zeiss AxioPlan2, Nikon Ti, or a V3 Delta Vision Elite 165 (Applied Precision Inc., a GE Healthcare Company, Issaquah, USA) microscope, with 1661.4 NA phase-contrast objectives. Images were analyzed using the MicrobeJ plug-in 167 for ImageJ (11). The cell circularity $\left(4 \pi \times\right.$ area / perimeter $\left.{ }^{2}\right)$ was measured on the 168 interpolated contour generated using the particle medial axis and the specified value 
169 of the width measured along the medial axis. Circularity values range between 0 and

170 1. Cell elongation was calculated as the inverse of the circularity.

171 For live cell imaging, a CellASIC ONIX microfluidics system (with B04A-03 plates),

172 was used with a Nikon Ti inverted microscope equipped with an incubated stage

$173\left(42^{\circ} \mathrm{C}\right)$. The four flow chambers were initially washed with $1 \mathrm{mg} / \mathrm{mL}$ Bovine Serum

174 Albumin (BSA) in phosphate-buffered saline followed by rinsing with $18 \%$ BSW at 5

175 psi for 5 min. Cells of $H$. volcanii (H98 + pTA962), freshly cultured on the agar

176 medium indicated with each movie, were resuspended in the respective liquid medium

177 (pre-warmed to $42^{\circ} \mathrm{C}$ ), and then loaded into the chambers as per the manufacturer's

178 instructions, and the chambers were perfused with medium at 2 psi for $20 \mathrm{~h}$, with

179 time-lapse images recorded at $10 \mathrm{~min}$ intervals.

180 Coulter cytometry

181 Culture samples were diluted (1:1000) with 18\% BSW, and then analyzed with a

182 Multisizer 4 Coulter cytometer (Beckman-Coulter) as described previously (6).

183 Western blotting

184 Western blotting was carried out as described previously (6).

185 


\section{Results}

187 It was previously shown that, in soft agar assays of cellular swimming motility, $H$. 188 volcanii cells change from a discoid (plate) shape into a rod shape in a CetZ1189 dependent manner (6). However, the soft-agar motility assay is not a robust method 190 for ongoing studies of cell-shape differentiation in $H$. volcanii as the soft agar is 191 difficult to separate from the cells, cell yields are low, and the distribution of shapes 192 observed is sensitive to the site of sampling from the soft agar (6). We and others 193 have noticed that $H$. volcanii can form regularly-shaped rods in the early stages of 194 culture, from around the time the culture transitions from lag to log phase, and then 195 cells revert to the irregular discoidal 'plate' cells later in log phase (12). As shown in 196 Fig. 1, cells obtained from colonies grown on agar medium are initially plate-shaped 197 upon resuspension in fresh $\mathrm{Hv}-\mathrm{Ca}$ liquid medium, but after overnight shaking 198 incubation (to an $\mathrm{OD}_{600}$ of 0.05 ) cells were predominantly rod-shaped. With further 199 incubation, until the culture reached $\mathrm{OD}_{600}$ of 0.3 , the rod-shaped cells had started to 200 disappear and by 0.9 the culture predominantly contained the plate-shaped cells (Fig.

201 1). Early culture cells had similar morphology to motile cells withdrawn from motility 202 agar (6), and were recently shown to be motile and suitable for direct observation of 203 swimming (12).

204 The $\mathrm{OD}_{600}$ where rods could be observed and then returned to plates varied between 205 cultures, possibly due to differences in the size of starting inoculum and the times 206 cultures take to reach equal culture density. We have also noticed widely varying 207 growth yields of $\mathrm{H}$. volcanii in $\mathrm{Hv}-\mathrm{Ca}$ medium prepared in different laboratories or 208 with different sources of reagents, and the extent and timing of early-culture rod 209 formation is generally sensitive to different media sources (e.g. we observed that one 210 lower-grade source of $\mathrm{NaCl}$ inhibited the early-culture rod development that was seen 211 in all other media tested). These issues make reproducing certain experiments difficult 212 between laboratories using different reagents. Such considerations are pertinent when 213 nutrition, metabolism, stress-response or whole-organism ('omic') projects are 214 planned.

215 The supply of trace elements in the medium was also observed to have dramatic 216 effects on growth and morphology, as described further below. The results shown in 
217 Fig. 1 were obtained with Hv-Ca medium without supplemental trace elements, but 218 growth in this culture was satisfactory as cells must have had sufficient supply of essential trace elements in this medium. However, such growth was not always observed with other reagent sources. We therefore sought to understand and standardize the conditions required for optimal growth and rod development; this led

222 to the identification of previously uncharacterized morphological responses of $H$. 223 volcanii.

\section{H. volcanii growth and morphology in nutrient-limited batch culture}

225 The commonly used media for $H$. volcanii, $\mathrm{Hv}-\mathrm{Ca}$ and $\mathrm{Hv}-\mathrm{YPC}$, consist of 226 macronutrients for heterotrophic growth in a base of $18 \%(\mathrm{w} / \mathrm{v})$ buffered primary sea227 water salts (10). Hv-Ca is a semi-defined medium with Casamino acids as the carbon 228 and energy source, and typically provides a slower growth rate and yield than the complex Hv-YPC medium, which contains Yeast extract, Peptone and Casamino

230 acids added to the $18 \%$ salts base. Both media are known to produce different 231 morphotypes in the same culture-primarily a mix of rods and plate cells, with some 232 other more unusual shapes also sometimes seen.

233 Since these media are normally not supplemented with micronutrients, beyond what 234 may be present as contaminants in the media reagents (10), and a range of metal ions 235 or other elements are typically required by cells at low or moderate concentrations to 236 act as essential cofactors in enzymatic reactions, we added a solution of eight 237 compounds as a source of micronutrients to the $\mathrm{Hv}-\mathrm{Ca}$ medium. This was based on 238 common requirements for microorganisms, and contained the elements $\mathrm{Fe}, \mathrm{Zn}, \mathrm{Cu}$, 239 Co, Mn, Ni, Mo and B ('trace elements', TE; see Methods). A vitamin solution (10) 240 was also added. The new medium (named "Hv-Cab", for the 'b' variant of Hv-Ca) 241 substantially improved the growth rate and yield, and their reproducibility, in $H$. 242 volcanii cultures, which was due primarily to the inclusion of the TE solution, as 243 described below.

244 To identify any growth or morphological changes associated with omission of the 245 primary nutrient sources in $\mathrm{Hv}-\mathrm{Cab}$, mid-log $H$. volcanii cells were transferred to 246 specific nutrient-omitted media, which were prepared with high-purity reagents to 247 minimize contaminating sources of trace elements or substances that might inhibit 
248 growth or development. Growth and cell morphology were monitored over three 249 successive passages (by dilution into fresh media) under each of these nutrient250 omitted conditions. Transfer to media without the carbon and energy source, 251 Casamino acids (CAA), caused cells to rapidly cease growth, as expected (Fig. 2A, 252 maroon data-points). Exclusion of the vitamins had a minor effect on the maximum $253 \mathrm{OD}_{600}$ that was consistent throughout the three successive culture passages, but had no 254 effect on cell shape (Fig. 2A-C). However, cultures without supplementary TE 255 showed successively greater reductions in growth rate and yield with each passage, 256 such that by the third passage growth was very poor, and the culture barely reached a 257 maximal $\mathrm{OD}_{600}$ of $\sim 0.3$ (Fig. $2 \mathrm{C}$ ).

258 Cells were observed by phase-contrast microscopy at day 5 (stationary phase) after all 259 three of the culture passages, and the cell shapes were visualized and analyzed by 260 automated measurement of the circularity of cell outlines; these data were then plotted 261 as histograms of cell circularity, expressed as a percentage compared to a perfect 262 circle (Fig. 2D-F). The stationary phase Hv-Cab cultures contained almost exclusively 263 plate-shaped cells that had near-circular cell outlines, similar to late log cultures. 264 However, the TE-depleted cultures showed striking cell elongation, which became 265 more extensive in each successive passage (Fig. 2H). Cells depleted of TE showed 266 complex features accompanying the extensively elongated rod shapes, including rods 267 that showed variable widths along their lengths, and some cells exhibiting substantial 268 polar tubules of variable length and width (Fig. 2H).

269 Improved growth of $H$. volcanii in TE-supplemented complex and semi-defined $270 \quad$ media

271 Previous studies have shown that in complex medium, such as Hv-YPC (10), 272 elongated and irregular cell morphologies can exist together with the plate-shaped 273 cells $(1,3)$. Trace elements are expected to be present in Hv-YPC, originating 274 primarily from the Yeast extract. We next investigated the possibility that trace 275 elements may yet be somewhat limiting for $H$. volcanii in Hv-YPC, giving rise to the 276 elongated forms seen in this medium, by supplementing Hv-YPC with the TE 277 solution. By analogy to the Hv-Cab media terminology introduced above, we refer to 278 the TE-supplemented version of Hv-YPC medium as Hv-YPCab. 
279 We initially took mid-log and stationary-phase cells grown in Hv-Cab medium, and

280 then washed and resuspended cells from both starters in Hv-YPC and Hv-YPCab, and

281 then monitored growth in microtiter-plates, to observe how well the cultures grew

282 under these new conditions. The growth rate and yields were noticeably greater in Hv-

283 YPCab medium compared to Hv-YPC (Fig. 3A). The Hv-YPCab culture grew well

284 even when started by inoculation with a stationary phase culture, compared to the Hv-

285 YPC culture inoculated with a mid-log starter, suggesting that the maintenance of

286 supplemental trace elements in the media is useful for minimizing the lag phase and

287 maximizing growth rates and yields of $H$. volcanii.

288 We also monitored growth and morphology of cells in standard tube-based batch289 cultures. Consistent with the results of Fig. 3A, cultures recommenced growth 290 substantially faster with Hv-YPCab medium, compared to the Hv-YPC medium (Fig. 291 3B). Similar trends were obtained with experiments based on the Hv-Ca medium (Fig. 292 3C), although growth was very poor, which was attributed to the lack of trace 293 elements in this medium made with high-purity reagents. Microscopy of late-stage 294 culture samples (at $40 \mathrm{~h}$ ) revealed that cells were almost uniformly the plate 295 morphotype in Hv-YPCab and Hv-Cab (Fig 3D, ‘+TE' panels), and showed none of 296 the elongated rods or filaments with tubules previously noted in non-TE297 supplemented medium (Fig. 3D, -TE panels). The cell size also appeared substantially 298 smaller in both TE-supplemented media compared to the original media (Fig. 3D). An 299 additional control culture, supplemented with the EDTA only showed that culture 300 growth, cell size and morphology were unaffected compared to Hv-YPC alone, 301 demonstrating that the cells were reacting specifically to the concentration of the 302 trace-element compounds that differ between the original and the supplemented media 303 (Fig 3D). Finally, we also observed a substantial difference in the morphology when 304 comparing cells in Hv-YPC and Hv-Ca (-TE cultures, Fig. 3D); the more elongated 305 cells observed in the Hv-Ca culture were attributed to the more severe TE starvation 306 in this medium (see Fig. 2).

307 Development of $H$. volcanii rods during an early stage of growth in culture

308 Based on the above results, the new supplemented media were expected to improve 309 consistency and reproducibility of the motile-rod development that occurs during 
310 early-culture. It was expected that the elongated/tubulated rod cell types specifically seen during TE-limitation would be absent, thus improving uniformity of the rods seen in early-culture growth. In order to thoroughly characterize the cell morphology

313 development of $H$. volcanii during the batch-culture growth cycle with the new media, 314 we inoculated cultures to a fixed cell density from consistently-prepared starter 315 cultures, and then monitored culture growth rate and cell morphology at regular 316 timepoints. H. volcanii was initially cultured for four days on the appropriate solid 317 media to allow colony development, and then sufficient colonies were picked and 318 resuspended in the equivalent liquid medium, and the $\mathrm{OD}_{600}$ was adjusted to 0.05 to 319 start the culture at a measurable and reproducible density. Culture growth $\left(\mathrm{OD}_{600}\right.$ and cell number), cell volumes (Coulter cytometry) and morphology (microscopy) were monitored over time, both with or without supplemental TE (Fig. 4).

In both Hv-Cab and Hv-YPCab media, cell volume and culture growth $\left(\mathrm{OD}_{600}\right)$ increased from the start of the experiments (Fig. 4B, 4D) and the cell number began to noticeably increase after $\sim 6 \mathrm{~h}$ delay (Fig. $4 \mathrm{C}$ ). This corresponded approximately to the onset of rod development, as seen in the representative images of the cells (Fig. 4E), and the quantitative analysis of cell elongation (Fig. 4A); in both media (+TE), a peak of rod development was observed commencing $~ 7-8 \mathrm{~h}$ after inoculation, reaching a maximum mean cell elongation after $\sim 10 \mathrm{~h}$, and then from $13 \mathrm{~h}$ onwards, plate cells were again almost exclusively seen (Fig. 4a, +TE). Similar results were obtained with cultures of the H26 (+ pTA962) strain inoculated from either agar-based colonies or 331 late-stage liquid cultures (Fig. S1). We also found that early-culture rod development required inclusion of the plasmid, which is based on the natural pHV2 plasmid from

333 H. volcanii and complements the uracil-auxotrophy of $\mathrm{H} 26$, indicating the importance 334 of plasmid inclusion or prototrophy in these assays (Fig. S1). Comparison of results 335 obtained with the different media showed that the peak of rod development was 336 greater in Hv-YPCab than in Hv-Cab, indicating a higher proportion or longer rods in 337 Hv-YPCab (compare the left-hand two panels in Fig. 4E, $11 \mathrm{~h}$ ). The timing of rod 338 development during the first few generations and subsequent conversion back to 339 plates approximately corresponded to the timing of an increase (growth) and then a 340 decrease (division) in the mean cell volume, respectively (Fig. 4B).

341 The effects of TE-starvation during the above colony-resuspension assay were also 
342 investigated. Cultures were first grown into mid-log phase in either $\mathrm{Hv}-\mathrm{Cab}$ or $\mathrm{Hv}-$

343 YPCab medium (i.e. +TE), and then they were separated from the medium by

344 centrifugation, washed (media -TE) and plated onto the respective -TE agar medium

345 (Hv-Ca and Hv-YPC). After growth on the agar, cells were resuspended in -TE liquid

346 medium. Cells resuspended from colonies were initially plate-shaped (Fig. 4A, 4E -

347 TE), even though there was a clear limitation of trace elements in the agar, detected

348 by very poor growth of colonies on the Hv-Ca agar. As expected, the Hv-Ca (-TE)

349 liquid cultures showed very poor overall growth, whereas Hv-YPC (-TE) cultures

350 grew quite well (Fig. 4C, 4D). Cells became noticeably elongated by 5 h (Hv-YPC, -

$351 \mathrm{TE})$ or $8 \mathrm{~h}$ (Hv-Ca, -TE) of incubation, and they continued to show substantial

352 elongated pleomorphology and increased cell volume for at least $20 \mathrm{~h}$, particularly in

353 the severe starvation conditions (Hv-Ca, -TE) (Fig. 4A, 4B, 4E).

354 Thus, as may be expected based on the poor growth and marked elongation responses

355 seen with severe TE starvation (Fig. 2), the transient appearance of the regularly-

356 shaped rod cell type seen in the optimally-growing cultures (+TE) was not clear

357 during these -TE assays. This emphasizes the importance of using complete, TE-

358 supplemented media for obtaining consistent results in the regular rod development

359 assays, and offers a standardized way of performing TE-starvation when those cell

360 types are required.

\section{Reversibility and specificity of the cell elongation response to TE-starvation}

362 To investigate the reversibility of the TE-starvation phenotype in the Hv-Ca cultures,

363 TEs were added at $15 \mathrm{~h}$ incubation after colony resuspension either by direct addition

364 of the TE stock solution, or by dilution of the Hv-Ca culture (1:100) into Hv-Cab, Hv-

365 Cab with 10x concentrated TE, or Hv-Ca (-TE) as a control. In all three media

366 conditions containing additional TE, the cultures reverted to almost exclusively the

367 plate cell morphotype at $12 \mathrm{~h}$ (Fig. 5). This is a similar duration required for the

368 appearance of elongated cells upon colony resuspension in $\mathrm{Hv}-\mathrm{Ca}$ (Fig. 4A, -TE), and

369 shows that the effect of TE-starvation on morphology is readily reversible by resupply

370 of the trace element nutrients. It is concluded that $H$. volcanii undergoes substantial

371 cell elongation (and increased tubule development) in response to trace-element

372 starvation, but not during starvation associated with stationary phase (+ TE) (Fig. 2-5, 
373 S2).

374 The potential effects of individual components of the TE solution on $H$. volcanii cell

375 shape were screened by testing a series of five TE solutions omitting either the Co,

$376 \mathrm{Cu}, \mathrm{Fe}, \mathrm{Mn}$ or $\mathrm{Zn}$. None of these individual 'drop-out' TE media matched the degree

377 of cell elongation observed when all TE were omitted (Fig. S3), suggesting that the

378 combined effect of multiple depleted elements causes the large change in cell shape

379 seen in the -TE conditions. The Co and Fe drop-out media appeared to cause the most

380 noticeable (although moderate) cell elongation response compared to the other metal

381 drop-out media (Fig. S3).

382 Microfluidic flow-chamber visualization of $\mathrm{H}$. volcanii cellular development

383 We next sought to directly visualize morphological development during the early log 384 growth phase in liquid culture. Cells resuspended from colonies were loaded into 385 incubated microfluidic chambers for time-lapse microscopy. Time-series of rod-cell 386 development in the media (+TE) are shown in Fig. 6A and 6B, and the complete time387 series are available in Movies S1 and S4. These data show that plate cells can initially 388 grow and divide as plates, and then most cells transition into rods as they grow and 389 divide over several generations. We observed that rods persisted during incubation in 390 the microfluidic culture system (particularly evident in Hv-YPCab), compared to the 391 temporally quite narrow peak of rods seen in batch culture (Fig. 4A). The continual 392 flow of fresh medium while cells are attached to the flow channels may therefore 393 create signals that maintain rod morphology.

394 Microfluidic cultures with media lacking the trace elements revealed striking 395 morphological responses (Fig. 6C, 6D, 6E, and Movies S2, S3 and S5), including 396 some extensively elongated cells exhibiting highly asymmetric division (Fig. 6C, 6E), 397 and the generation of some bizarre irregular shapes (Fig 6D). This supported the batch 398 culture results suggesting that formation of regularly shaped rods in early log phase is 399 promoted in optimal growth conditions (in the presence of TE), whereas irregularly 400 shaped and elongated shapes are associated with growth in the absence of TE and 401 hence suboptimal growth conditions. Interestingly, tubules appeared to be generated 402 by a combination of localized narrowing and elongation (tubulation), often in the mid403 cell region of a cell, creating an approximate dumbbell-like appearance, followed by 
404 division near one end of the narrowing. We also saw that occasionally the narrow

405 zone can re-widen, in a peristaltic-like wave along the cell (Movie S5).

406 Requirement of tubulin-like protein CetZ1 for elongated shapes and tubule 407 formation

408 In order to test whether the morphological changes require an internal cytoskeleton, 409 we investigated the importance of the CetZ1 tubulin-like cytoskeletal protein in the 410 cell shape changes. CetZ1 was previously found to be required for rod-cell 411 development when cells become motile (6). A CetZ1-knock-out strain was subjected 412 to the assays defined in Fig. 4, to determine if rod-shape or tubule development were 413 affected under these conditions. In early-culture rod development assays, the $\Delta$ cet $Z 1$ 414 strain failed to form rods at $10 \mathrm{~h}$ post-resuspension in $\mathrm{Hv}-\mathrm{Cab}$ or $\mathrm{Hv}-\mathrm{YPCab}$, 415 compared to clear rod formation in the wild-type control (Fig. 7A). Furthermore, both 416 the elongated rods and the tubules observed in wild-type cells in the -TE conditions 417 (Fig. 4) were not evident in $\Delta$ cetZl cells (Fig. 7A). We also used Coulter cytometry to 418 assess cell volumes of wild-type and CetZ1 knock-out strains. They showed very 419 similar cell-size distributions in both media (15 h post-resuspension; Fig. 7C), despite 420 the different shapes (Fig. 7A). However, both strains showed larger cells on average 421 in $\mathrm{Hv}-\mathrm{Ca}$ (-TE) than in $\mathrm{Hv}-\mathrm{Cab}$, demonstrating that cell size (regulation of cell 422 division) also responds to trace-element starvation.

423 We confirmed the expected absence of CetZ1 protein in the knock-out strain by 424 Western blotting. We also tested whether the inclusion of TE influences cellular 425 CetZ1 concentration, but observed no substantial difference (Fig. 7B). To verify that 426 CetZ1 function is necessary for the rod and tubule formation, we expressed a 427 dominant-inhibitory mutant (CetZ1.E218A) from a plasmid in a wild-type genetic 428 background and then observed cell shapes. The E218A mutation in the GTPase 429 active-site strongly inhibits CetZ1 function via hyper-stabilization of localized 430 polymers, and was used previously for verification of CetZ1's involvement in rod 431 development in motility soft-agar (4). We found that expression of cetZ1.E218A 432 blocked rod cell and tubule formation in the wild-type strain background (Fig. 7A), 433 confirming its dominant influence on cell shape and the requirement for functional 434 CetZ1 in trace-element-induced rod and tubule generation. Finally, we found that 
435 there were no detectable differences in growth rate or yield when comparing the wild-

436 type, $\triangle$ cetZ1 and cetZ1.E218A-expression strains in Hv-YPC medium lacking TE

437 (Fig. S4), showing that the morphology differences are not associated with substantial 438 changes in growth during moderate TE limitation. 
439

440

441

442

443

444

445

446

447

448

449

450

451

452

453

454

455

456

457

458

459

460

461

462

463

464

465

466

467

468

469

\section{Discussion}

We report improved $H$. volcanii growth, in both rich and semi-defined media, by inclusion of eight supplementary trace elements (TE) as well as the identification and characterization of culture-dependent morphological changes that are more reproducible in the presence of TE.

Our surprising observation that TE-supplementation can improve growth and shape uniformity even in the complex medium containing yeast-extract made with highpurity salt, buffer, and water suggests that the optimal growth of $H$. volcanii requires the presence of relatively high levels of certain 'trace' metals. Previous $H$. volcanii media have been prepared with a trace elements solution containing $\mathrm{Mn}, \mathrm{Zn}, \mathrm{Cu}$ and Fe (13), but these are unlikely to represent all the minor elements utilized by $H$. volcanii (Fig. 3 and Fig. S3), and, furthermore, this four-element solution has only been widely adopted for use in minimal medium (10). More recent reports on traceelement supplementation in $H$. volcanii media have used six (14) of the elements used in the current study, and all eight elements have been used with synthetic medium (15), although in differing proportions. However, these studies did not report the influence these elements had on growth and morphology. Relatively high but variable metal ion concentrations occur in hypersaline lakes including the Dead Sea (the origin of $H$. volcanii DS2), which is especially rich in manganese $(\sim 100-150 \mu \mathrm{M})(1,16)$.

Why would trace-element limitation trigger the development of $H$. volcanii elongation or tubules? Some of the factors that are related to morphological changes in bacteria are nutrient availabiliy, extracellular signalling, cell attachment and biofilm formation, motility/chemotaxis, predation and polar differentiation $(17,18)$. For example, cells, starving for multiple nutrients results in cell shape changes in a marine Vibrio species, where the pleomorphic cell populations stay viable for extended periods (19). In Caulobacter crescentus, phosphate starvation triggers stalk extension, which is thought to increase surface area for nutrient uptake $(20,21)$. Why $H$. volcanii changes cell shape during trace element starvation is not yet clear, however, the observation that these cells also get larger (Fig. 4B), thus reducing surface area-tovolume ratio, suggests that the overall response might do more than improving nutrient-uptake. 
470 Our study also characterized the timing and conditions for $H$. volcanii rod

471 development during the early stages of growth in dilute liquid cultures including 472 supplemental TE. These rods were regularly-shaped and appear similar to the motile 473 rods taken from soft agar (6). Indeed, early log cells were recently shown to be motile 474 and suitable for direct observation of swimming (12). H. volcanii cell shape changes 475 are also highly sensitive to factors other than nutrient availability; for example, we 476 found that early-culture rod formation in the high-purity reagent medium, requires the 477 presence of an $H$. volcanii plasmid, pTA962 (Fig. S1), which contains the endogenous 478 pHV2 replication functions and marker genes (pyrE2, hdrB) that complement 479 auxotrophic strains. A previous study noted a similar effect of the plasmid on the 480 prevalence of rods (7), and we have also observed a slight decrease in cell size 481 (unpublished data). While the plasmid does not appear to significantly affect growth 482 rate (9), the results suggest that $H$. volcanii cell size and shape are influenced by 483 auxotrophic status, plasmid replication and other functions, possibly through their 484 impacts on metabolism. Other cellular responses, including the development of 485 motility in soft agar, appear to be less sensitive to some of these effects (6). These 486 findings emphasize the general importance of using strains that are genetically 487 complemented to a wild-type status; simply supplying the required factors for 488 auxotrophic growth is inadequate to avoid diverse effects on these cells.

489 The rod development that occurs during the early growth stages of a culture after 490 resuspension or dilution of $H$. volcanii in fresh liquid medium is consistent with the 491 sensing of nutrients or diluted biotic signals such as secreted quorum-sensing 492 molecules. Consistent with this, we also observed cells retaining their rod shapes for 493 extended periods when cells are exposed to a continuous flow of fresh medium. 494 However, cells might also sense surface attachment and shear forces that differ 495 between the flow chambers and liquid batch cultures. We also observed that cell 496 shapes in agar surface colonies differed greatly from cell shapes in liquid cultures 497 made with medium that was otherwise the same (both -TE), suggesting that the 498 morphological effect of growth on agar (producing the plate morphotype) is dominant 499 over the influence of trace element limitation, and could reflect sensing of different 500 chemical or physical signals in the colonies. Since $H$. volcanii is capable of quorum 501 sensing (22) and mechanosensing (23), further studies could ascertain how these 
502 processes might trigger changes in cell shape.

\section{Author contribution statement}

504 Designed research: RTdS, MP, IGD. Contributed data: MFAH, MP (Fig. 1), RTdS

505 (Fig. 2-7, S2-3, Movies S1-S5), DAP, HJB (Fig. S1, S4). Interpreted data: RTdS, MP,

506 DAP, IGD. Wrote the manuscript: RTdS, IGD.

\section{Conflict of interest statement}

508 The authors declare that there are no conflicts of interest.

\section{Acknowledgements}

510 This work was supported by the Australian Research Council (FT160100010 and

511 DP160101076). M.P. and M.F.A.-H. were supported by the National Science

512 Foundation grant 1817518. We are grateful for use of the equipment and support from

513 the UTS Microbial Imaging Facility staff Louise Cole, Michael Johnson and Chris

514 Evenhuis, and Solenne Ithurbide and Stephen Vadia for comments on the manuscript. 
517 1. Mullakhanbhai MF, Larsen H. Halobacterium volcanii spec. nov., a Dead Sea 518 halobacterium with a moderate salt requirement. Arch Microbiol. 1975;104(3):20751914.

520 2. Bisson-Filho AW, Zheng J, Garner E. Archaeal imaging: leading the hunt for 521 new discoveries. Molecular biology of the cell. 2018;29(13):1675-81.

522 3. Delmas S, Duggin IG, Allers T. DNA damage induces nucleoid compaction

523 via the Mre11-Rad50 complex in the archaeon Haloferax volcanii. Molecular

524 Microbiology. 2013;87(1):168-79.

525 4. Liao Y, Ithurbide S, de Silva RT, Erdmann S, Duggin IG. Archaeal cell

526 biology: diverse functions of tubulin-like cytoskeletal proteins at the cell envelope.

527 Emerg Top Life Sci. 2018;2(4):547-59.

528 5. Chimileski S, Franklin MJ, Papke RT. Biofilms formed by the archaeon

529 Haloferax volcanii exhibit cellular differentiation and social motility, and facilitate

530 horizontal gene transfer. BMC Biol. 2014;12:65.

531 6. Duggin IG, Aylett CH, Walsh JC, Michie KA, Wang Q, Turnbull L, et al.

532 CetZ tubulin-like proteins control archaeal cell shape. Nature. 2015;519(7543):362-5.

533 7. Abdul Halim MF, Karch KR, Zhou Y, Haft DH, Garcia BA, Pohlschroder M.

534 Permuting the PGF Signature Motif Blocks both Archaeosortase-Dependent C-

535 Terminal Cleavage and Prenyl Lipid Attachment for the Haloferax volcanii S-Layer

536 Glycoprotein. J Bacteriol. 2015;198(5):808-15.

537 8. Abdul-Halim MF, Schulze S, DiLucido A, Pfeiffer F, Bisson Filho AW,

538 Pohlschroder M. Lipid Anchoring of Archaeosortase Substrates and Midcell Growth

539 in Haloarchaea. mBio. 2020;11(2).

5409 9. Allers T, Barak S, Liddell S, Wardell K, Mevarech M. Improved strains and

541 plasmid vectors for conditional overexpression of His-tagged proteins in Haloferax

542 volcanii. Appl Environ Microbiol. 2010;76(6):1759-69.

543 10. Allers T, Ngo HP, Mevarech M, Lloyd RG. Development of additional

544 selectable markers for the halophilic archaeon Haloferax volcanii based on the leuB

545 and trpA genes. Appl Environ Microbiol. 2004;70(2):943-53.

546 11. Ducret A, Quardokus EM, Brun YV. MicrobeJ, a tool for high throughput

547 bacterial cell detection and quantitative analysis. Nat Microbiol. 2016;1(7):16077.

548 12. Li Z, Kinosita Y, Rodriguez-Franco M, Nußbaum P, Braun F, Delpech F, et al.

549 Positioning of the Motility Machinery in Halophilic Archaea. mBio.

$550 \quad 2019 ; 10(3): \mathrm{e} 00377-19$.

551 13. Cline SW, Schalkwyk LC, Doolittle WF. Transformation of the

552 archaebacterium Halobacterium volcanii with genomic DNA. J Bacteriol.

553 1989;171(9):4987-91.

554 14. Hattori T, Shiba H, Ashiki K, Araki T, Nagashima YK, Yoshimatsu K, et al.

555 Anaerobic Growth of Haloarchaeon Haloferax volcanii by Denitrification Is

556 Controlled by the Transcription Regulator NarO. J Bacteriol. 2016;198(7):1077-86.

557 15. Maurer S, Ludt K, Soppa J. Characterization of Copy Number Control of Two

558 Haloferax volcanii Replication Origins Using Deletion Mutants and Haloarchaeal

559 Artificial Chromosomes. J Bacteriol. 2018;200(1).

560 16. Nissenbaum A. Minor and trace elements in Dead sea water. Chem Geol.

$561 \quad 1977 ; 19: 99-111$.

562 17. Young KD. Bacterial morphology: why have different shapes? Curr Opin 
563 Microbiol. 2007;10(6):596-600.

564 18. Justice SS, Hunstad DA, Cegelski L, Hultgren SJ. Morphological plasticity as 565 a bacterial survival strategy. Nat Rev Microbiol. 2008;6(2):162-8.

566 19. Stretton S, Danon SJ, Kjelleberg S, Goodman AE. Changes in cell 567 morphology and motility in the marine Vibrio sp. strain S14 during conditions of 568 starvation and recovery. FEMS microbiology letters. 1997;146(1):23-9.

569 20. Klein EA, Schlimpert S, Hughes V, Brun YV, Thanbichler M, Gitai Z.

570 Physiological role of stalk lengthening in Caulobacter crescentus. Commun Integr

571 Biol. 2013;6(4):e24561.

572 21. Wagner JK, Setayeshgar S, Sharon LA, Reilly JP, Brun YV. A nutrient uptake 573 role for bacterial cell envelope extensions. Proc Natl Acad Sci U S A.

$574 \quad 2006 ; 103(31): 11772-7$.

575 22. Megaw J, Gilmore BF. Archaeal Persisters: Persister Cell Formation as a

576 Stress Response in Haloferax volcanii. Front Microbiol. 2017;8:1589.

577 23. Le Dain AC, Saint N, Kloda A, Ghazi A, Martinac B. Mechanosensitive ion

578 channels of the archaeon Haloferax volcanii. J Biol Chem. 1998;273(20):12116-9. 


\section{$580 \quad$ Figures}

581

582
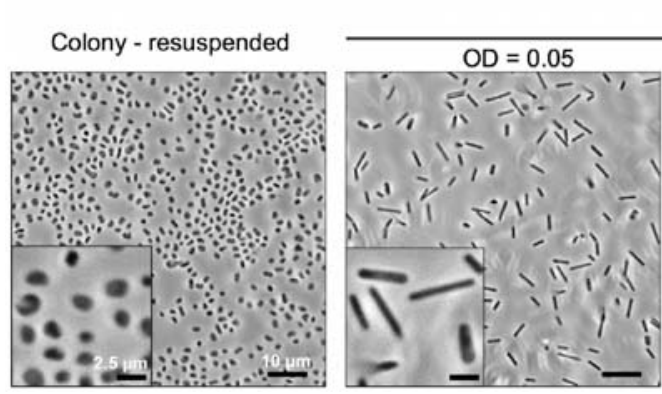

Liquid culture
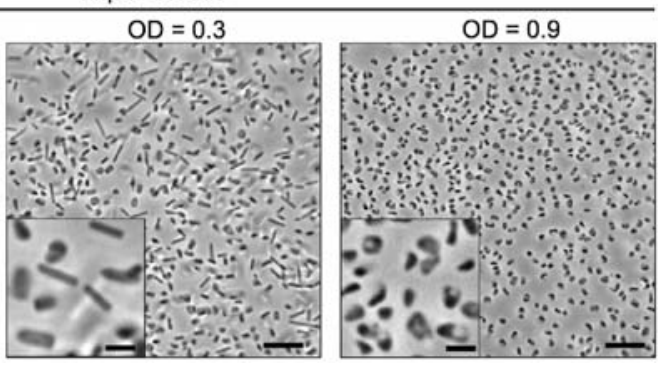

584

Figure 1. H. volcanii forms rods during the early stages of growth at low culture used to inoculate $\mathrm{Hv}-\mathrm{Ca}$ liquid medium (without additional vitamins or trace elements, but prepared with standard-purity reagents as the only possible source of trace elements). Cells from early-, mid- and late-log phase cultures $\left(\mathrm{OD}_{600} 0.05,0.3\right.$,

591 and 0.9 , respectively) were observed by phase-contrast microscopy. Scale bars are 10

592 

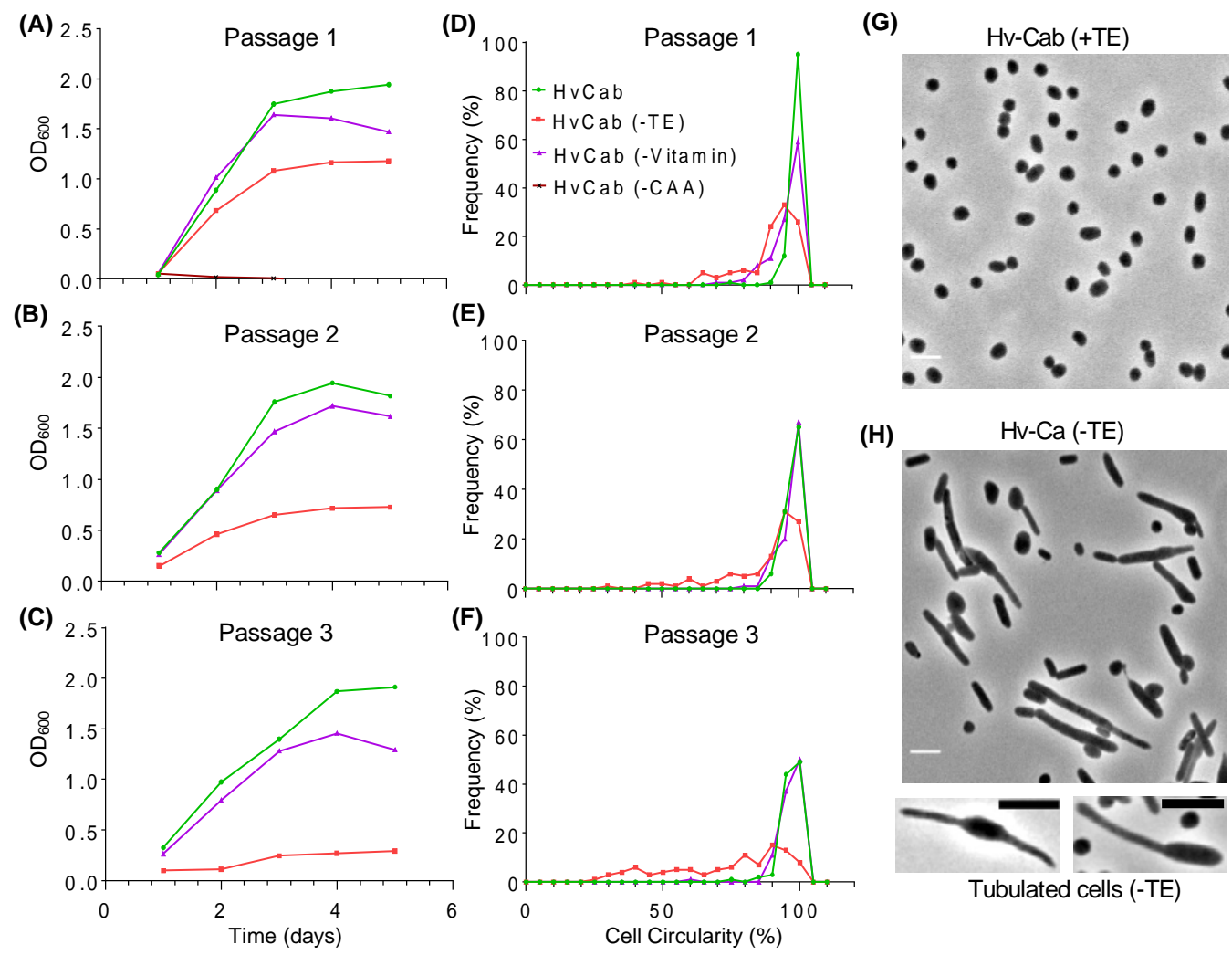

Figure 2. Effects of nutrient depletion on $\mathrm{H}$. volcanii growth and morphology in

596 liquid culture. Strain H98 + pTA962 was initially grown to mid-log phase in Hv-Cab $597\left(\mathrm{OD}_{600}=0.5\right)$, and then was washed 3 times by centrifugation at 5,000 rpm in a 598 microcentrifuge for $5 \mathrm{~min}$, followed by resuspension of the cell pellet in same volume 599 of $18 \%$ BSW, and diluted (1:100) into fresh Hv-Cab or in media excluded for one of 600 the major nutrient components, i.e. trace elements (TE, red data-points), vitamins 601 (biotin and thiamine, purple triangles), or Casamino acids (CAA, maroon triangles).

602 (A) Culture growth was monitored by measuring the $\mathrm{OD}_{600}$ over 5-days. Cells from 603 day-5 of the first dilution were then diluted (1:100) into the respective nutrient604 depleted media and incubated and monitored the same way for a further 5 days (B). 605 Cells from the second dilution (day-5) were again diluted (1:100) into the respective 606 nutrient-depleted media and then incubated for five more days (C). Cells imaged by 607 phase-contrast microscopy at day-5 (final data point) in each of the cultures (A-C) by 608 determining the circularity of cell outlines $(\mathrm{N}=100$, randomly selected cells), shown 609 as a corresponding histogram (D-F). G and $\mathrm{H}$ shows the phase-contrast images of 610 cells in (G) Hv-Cab (+TE) and (H) Hv-Ca (-TE) on day-5 of the third passage (a 611 representative image of the cells analysed in histogram F). TE depletion also resulted 612 in formation of polar tubules in some cells. The scale bars are $5 \mu \mathrm{m}$. 


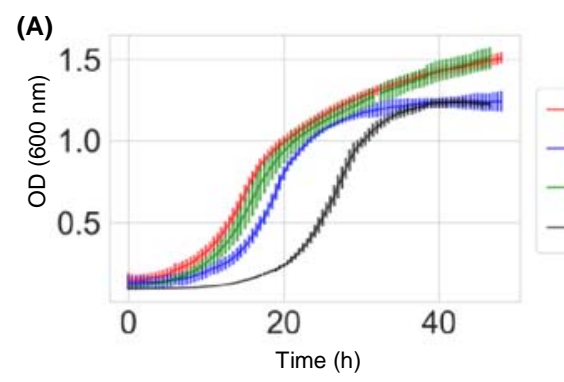

(B)

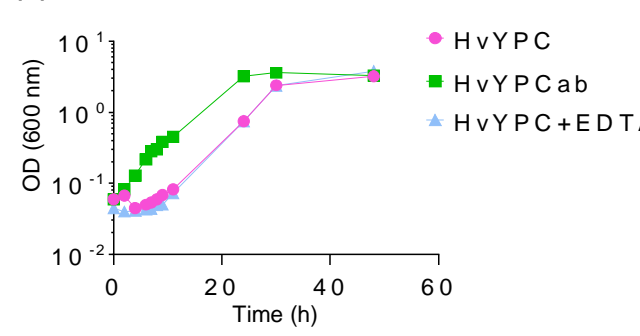

(C)

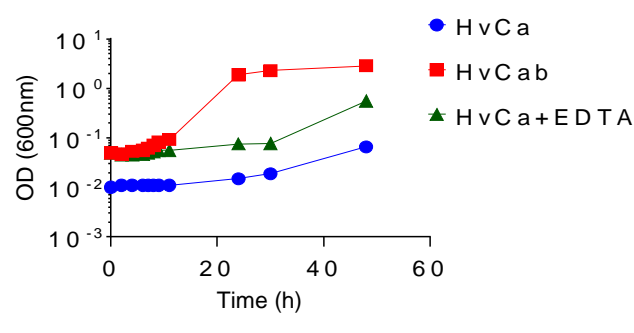

Figure 3. Media supplementation with a trace elements (TE) solution improves 618 growth and cell shape uniformity in $\boldsymbol{H}$. volcanii. (A) Growth curves in microtiter plate format. Two H. volcanii (H98 + pTA962) Hv-Cab batch cultures $(5 \mathrm{~mL})$ were started from separate colonies. The cells were taken at the mid-log phase (for 6 technical replicates) of one culture and at the stationary phase (for 3 technical replicates) of the other. The error bars indicate the standard deviation. (B) Growth curves in tube-culture format, started from steady mid-log cultures in the respective media, comparing Hv-YPC medium with the same media supplemented with the complete TE solution (i.e. Hv-YPCab), or with only the EDTA component of the solution (control). (C) Tube-culture experiments, as per panel (B) except based on $\mathrm{Hv}$-Ca medium. The Hv-Ca culture was started at $\sim 10$-fold lower cell density than the other two, owing to the relatively poor growth of the starter culture. (D) Cells sampled after $40 \mathrm{~h}$ (stationary phase) were imaged by phase-contrast microscopy. All scale bars represent $5 \mu \mathrm{m}$. 

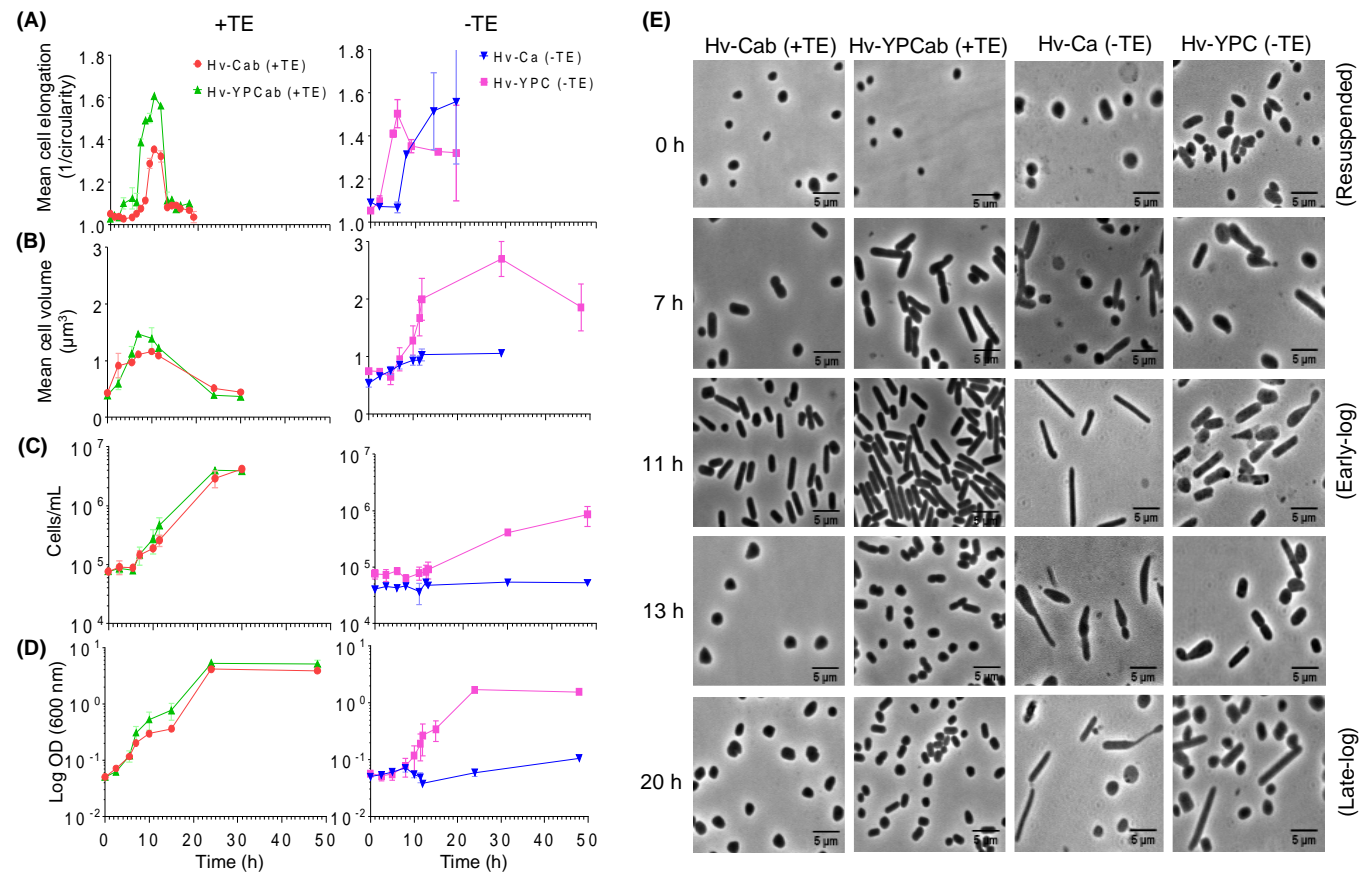

Figure 4. Assays for $H$. volcanii reversible morphological transitions during the growth cycle. Samples of $H$. volcanii (H98 + pTA962), grown in the indicated liquid media (after inoculation from colonies obtained from 4 days of growth on the equivalent solid agar medium), were withdrawn at time-points for microscopy (A)(E), Coulter cytometry determination of cell volumes (B) and counts (C), and $\mathrm{OD}_{600}$ measurements (D). Mid-log growth rates $\left(\mu\right.$ mean \pm SD) were: $\mathrm{Hv}-\mathrm{Ca}=0.045 \pm 0.011 \mathrm{~h}^{-}$ 1, Hv-Cab $=0.149 \pm 0.020 \mathrm{~h}^{-1}, \mathrm{Hv}-\mathrm{YPC}=0.141 \pm 0.008 \mathrm{~h}^{-1}$, and Hv-YPCab $=$ $0.206 \pm 0.003 \mathrm{~h}^{-1}$. The microscopy analysis in panel (A) shows mean cell elongation (expressed as inverse circularity; a value of 1 is a perfect circle and becomes greater as cells become more elongated) of 250 randomly selected cells at each time point. Datapoints shown represent the mean from two independent replicates, and error bars 
(B) $0 \mathrm{~h}$

Key (panels A and C):

(a) TEs were added directly to culture (b) Culture diluted into $\mathrm{Hv}-\mathrm{Cab}(+\mathrm{TE})$

(c) Culture diluted into $\mathrm{Hv}-\mathrm{Cab}(+10 \times \mathrm{XTE})$

(d) Culture diluted into $\mathrm{Hv}-\mathrm{Ca}(-\mathrm{TE})$

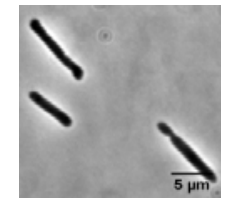

(A)

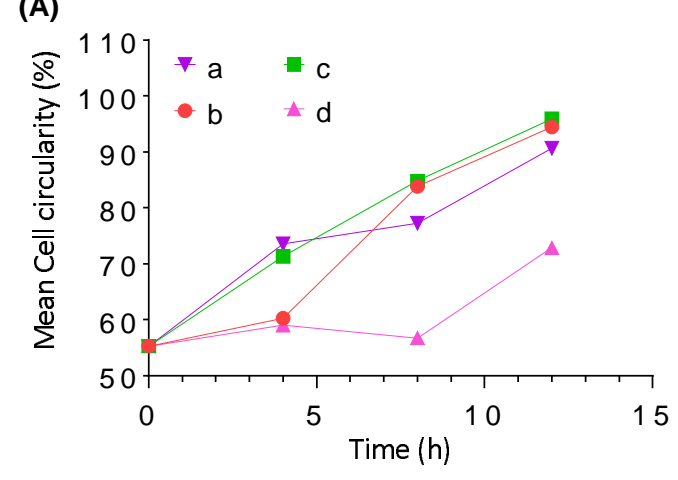

(C)

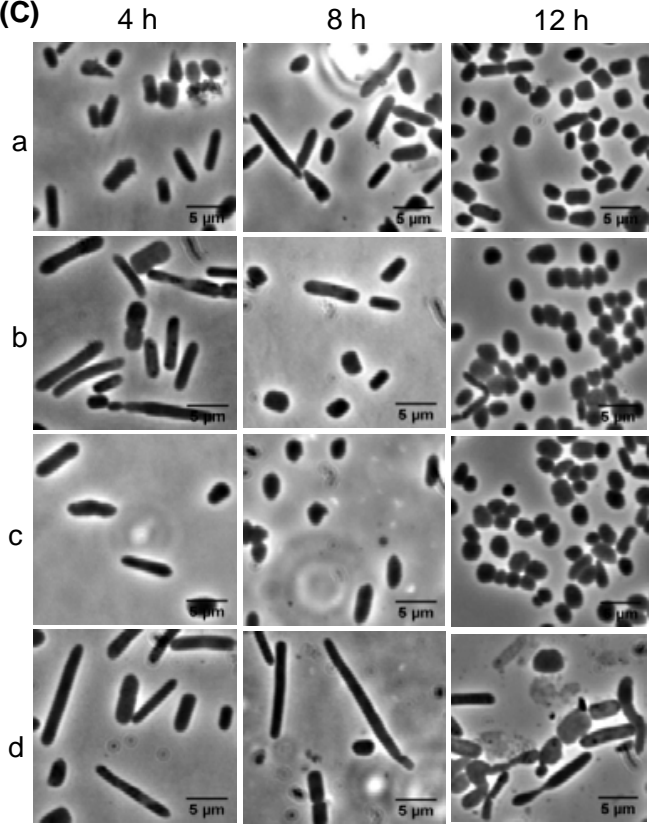

651 Figure 5. Reversal and specificity TE starvation. H. volcanii (H98 + pTA962)

652 colonies from Hv-Ca agar were resuspended in Hv-Ca liquid medium. After $15 \mathrm{~h}$ of 653 incubation, the culture was treated as indicated in the key, and then each new culture 654 was monitored by sampling at $3 \mathrm{~h}$ intervals for microscopy over 12 hours. (A) The 655 mean cell circularity (sample size $\mathrm{N}=150$ at each time point) versus time. (B) 656 Representative phase-contrast image of the cells from the Hv-Ca (-TE) culture at $15 \mathrm{~h}$, 657 immediately prior to introducing TE. (C) Images containing cells after $4 \mathrm{~h}, 8 \mathrm{~h}$ and 12 $658 \mathrm{~h}$ incubation under the conditions given in the key. Scale bars represent 5 microns. 
(A) $\mathrm{Hv}-\mathrm{Cab}(+\mathrm{TE})$

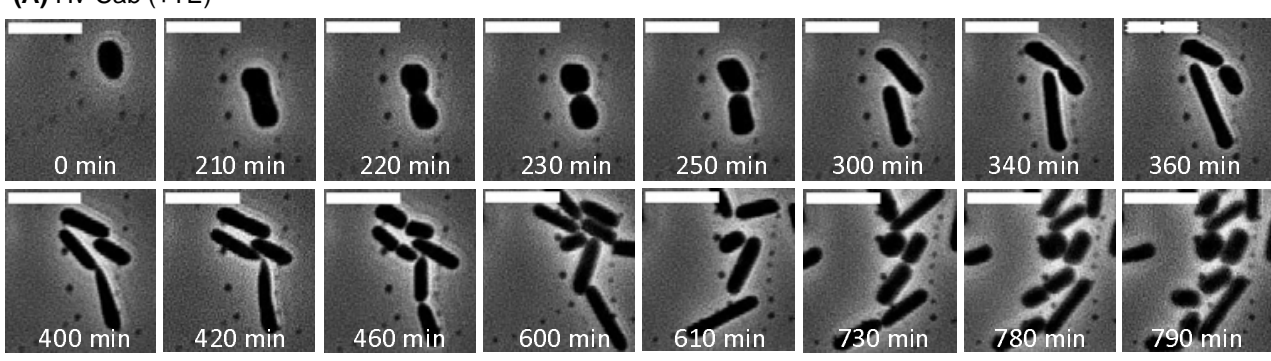

(B) Hv-YPCab (+TE)
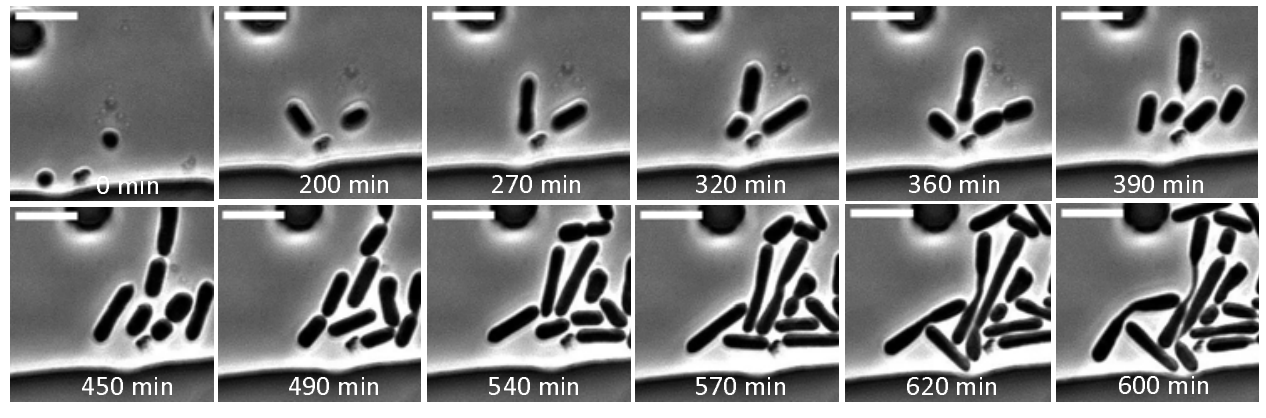

(C) Hv-Ca (-TE)

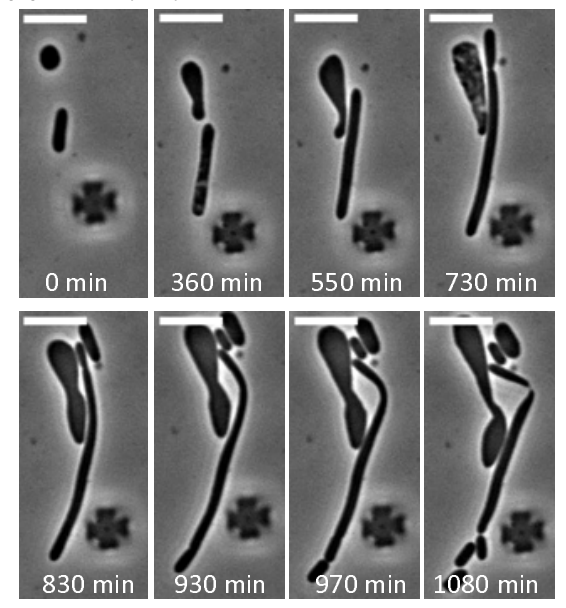

(D) Hv-Ca (-TE)

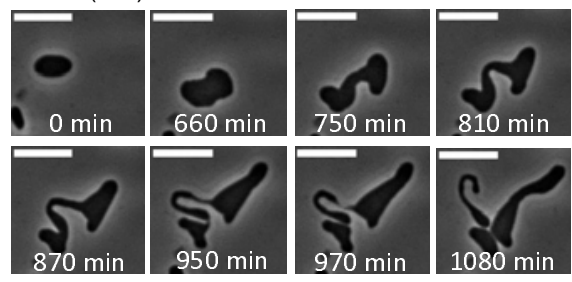

(E) Hv-YPC (-TE)

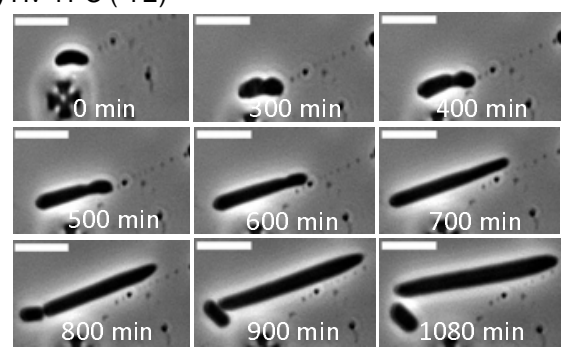

662 Figure 6. Time-lapse microscopy of $H$. volcanii $($ H98 + pTA962) with and without

663 trace elements. Microfluidic chambers were loaded with cells and phase-contrast 664 images were collected at 10 min intervals during perfusion ( 2 psi for $20 \mathrm{~h}$ ). (A) 665 Selected time frames showing initial growth after resuspension in Hv-Cab (+TE). (B) 666 Selected time frames during microfluidic culture in Hv-YPCab (+TE). (C) Selected 667 time frames showing the substantial cell elongation observed Hv-Ca (-TE) and a 668 highly asymmetric division event. (D) Another field of cells cultured in Hv-Ca (-TE) 669 showing slow growth and striking morphologies, including the generation of a cells 670 that shows a distinctive highly curved narrowing or tubule. (E) Selected images 671 during cell elongation in Hv-YPC (-TE). 
(A)

(A) $\mathrm{Hv}-\mathrm{Cab}(+\mathrm{TE}) \quad \mathrm{Hv}-\mathrm{YPCab}(+\mathrm{TE}) \quad \mathrm{Hv}-\mathrm{Ca}(-\mathrm{TE}) \quad \mathrm{Hv}-\mathrm{YPC}(-\mathrm{TE})$
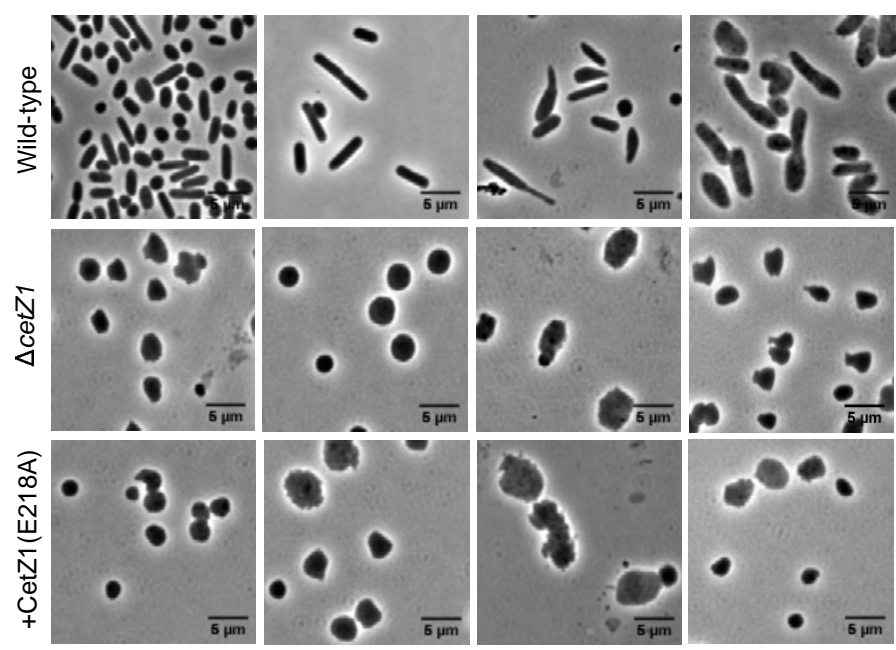

(B)

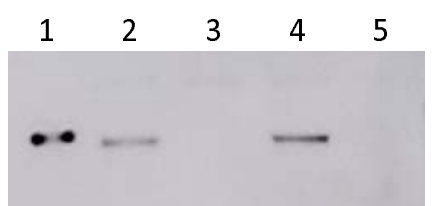

1) Pure CetZ1

2) Wild-type - Hv-Cab (+TE)

3) $\Delta$ cetZ1 - Hv-Cab (+TE)

4) Wild-type - Hv-Ca (-TE)

5) $\Delta$ cetZ1 - Hv-Ca (-TE)

(C)

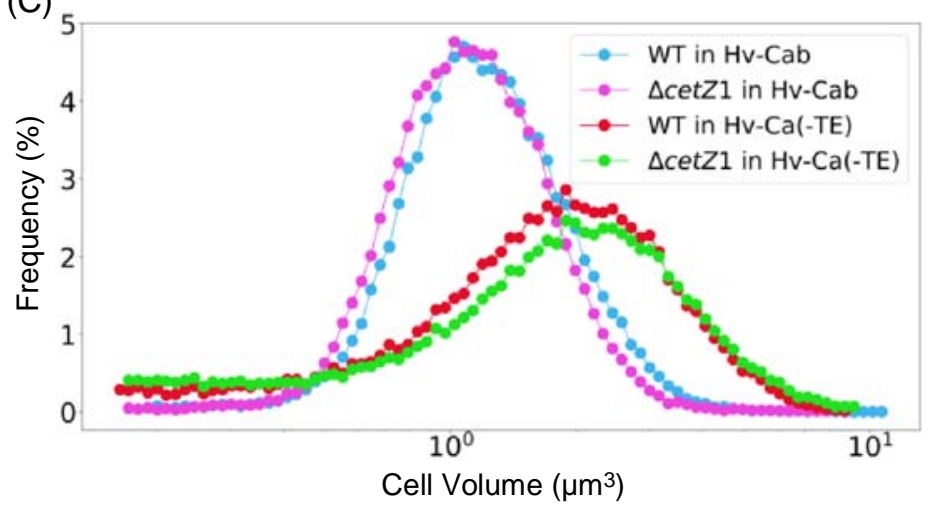

673 Figure 7. CetZ1 is essential for rod development during early-growth after 674 colony resuspension and during TE-depletion. (A) Phase-contrast images of wild675 type (H98 + pTA962), the CetZ1 knock-out (H98.4cetZ1 (ID59) + pTA962), and the point-mutant (H98 + pTA962-cetZ1.E218A) were recorded $10 \mathrm{~h}$ post-resuspension of colonies in the indicated media (including $1 \mathrm{mM}$ L-tryptophan, as the inducer for the P.tna promoter in pTA962). In $\Delta c$ cetZ1, or during expression of CetZ1.E218A (-TE conditions), cells failed to form rods or substantially elongate, and sometimes exhibited a ruffled cell shape, showing small protrusions and grooves. (B) Western blot probing CetZ1 in the wild-type and CetZ1 knock-out strains at $15 \mathrm{~h}$ postresuspension in the indicated media. (C) Coulter cytometry cell-volume distributions of the wild-type and CetZ1 knock-out strains at $15 \mathrm{~h}$ post-resuspension in $\mathrm{Hv}-\mathrm{Cab}$ and $\mathrm{Hv}-\mathrm{Ca}(-\mathrm{TE})$. 